children treated for ALL. In a study from the Institute of Child Health, University of London, children who received a second course of cranial radiotherapy for relapsing LL suffered from neurologic deficits, growth impairment, ventricular enlargement on MRI, and impairments of tests of verbal comprehension, attention, and memory. Girls were affected more than boys. (Ped Neur Briefs June 1994;8:47). Hypoplasia of the cerebellar vermis and cognitive deficits involving visual-spatial-motor coordination and memory were reported at 9 year follow-up in 13 children who received 24 Gy cranial radiation and intrathecal methotrexate at the University of New Mexico and centers in Canada. (Ped Neur Briefs Nov 1994;8:82). The late morbidity associated with cranial radiotherapy has been recognized for some time. The Children's Cancer Group study demonstrates that cognitive deficits may become evident as early as 9 months after treatment, even with more moderate levels of irradiation. Whole-brain radiotherapy for brain tumors may also result in significant IQ deficits in children treated before age 7 . (Progress in Pediatric Neurology II, 1994, p199).

\title{
LANGUAGE DOMINANCE WITH LEFT HEMISPHERE TUMORS
}

Hemispheric language dominance in 12 children with slow growing tumors near left hemisphere language areas was studied at the Cleveland Clinic, OH. Complex partial seizures began at or under 6 years of age. Tumor resection was performed at ages 5 to 20 years, an average of 8 years after seizure onset. Neurologic exam was normal in 11 patients. Ten $(83 \%)$ had amobarbital tests indicating left hemisphere language dominance, confirmed by cortical stimulation in 6 . Eleven with complete tumor resection were seizure-free at follow-up. Most had developmental neoplasms, often congenital. Paired $t$ tests found no differences between pre- and postoperative performance of language tests, and a tendency for improved language function after operation. (DeVos KJ et al. Language dominance in patients with early childhood tumors near left hemisphere language areas. Neurology February 1995;45:349-356). (Reprints: Dr Elaine Wyllie, Pediatric Epilepsy Program, The Cleveland Clinic Foundation, 9500 Euclid Ave, Cleveland, OH 44195).

COMMENT. Language dominance does not usually transfer to the contralateral hemisphere in young children with low-grade left frontal and temporal tumors. Successful tumor resection is accomplished by using cortical stimulation studies and sparing of the language areas in close proximity to the tumor.

\section{NEUROMUSCULAR DISORDERS}

\section{PYRIDOXINE-INDUCED SENSORY NEUROPATHY}

An 18-year-old man with seizures from birth was followed in the Department of Clinical Neurological Sciences, University of Western Ontario, London, and was found to have developed a sensory neuropathy by 2 years of age following treatment with pyridoxine in doses up to $2000 \mathrm{mg} /$ day. The initial seizure at birth responded to pyridoxine $150 \mathrm{mg} \mathrm{IV}$, after treatment with diazepam had failed. A sister had died in status epilepticus at age 9 days and had not received pyridoxine. Complex febrile seizures from 1 to 4 years, followed by recurrent afebrile convulsions, and at 13 years, complex partial seizures continued despite pyridoxine $2000 \mathrm{mg} /$ day, phenytoin, and phenobarbital. At 
18 years, following the addition of carbamazepine, seizures were controlled, and pyridoxine was decreased to $100 \mathrm{mg}$ daily. MRI showed left mesial temporal sclerosis. Nerve conduction studies at 2 years revealed absent sensory action potentials and normal motor conduction. Sural nerve biopsy showed severe, axonal, sensory neuropathy. At 18 years, vibration sense in the feet was absent, position sense was decreased in the toes, pain sensation was impaired to the midcalf and in the fingers, tendon reflexes were absent, and plantar responses were flexor. His gait was ataxic. Sural, peroneal and median sensory nerve action potentials were absent. The sensory neuronopathy diagnosed at 2 years had not progressed or remitted at 18 years. (McLachlan RS, Brown WF. Pyridoxine dependent epilepsy with iatrogenic sensory neuronopathy. Can I Neurol Sci February 1995;22:50-51). (Reprints: Dr RS McLachlan, University Hospital, 339 Windermere Rd, London, Ontario N6A 5A5).

COMMENT. The authors explain the failure of pyridoxine to completely control the seizures in this patient by a combination of pyridoxinedependent epilepsy with complex partial seizures due to mesial sclerosis. Unusually high doses of pyridoxine were prescribed in this patient.

Doses as low as $50 \mathrm{mg}$ /day have caused neuropathy when continued for months or years. Individual susceptibility is also a factor in the occurrence of this side effect.

\section{CRITICAL ILLNESS NEUROMUSCULAR DISEASE}

Four children with critical illness neuromuscular disease following prolonged dependency on a ventilator are reported from the Departments of Neurology and Pediatrics, West Virginia University Health Sciences Center, Morgantown, and the Department of Medicine (Neurology), Memorial University of Newfoundland, St John's, Canada. One patient, a 15-year-old boy with septic shock, required ventilatory support and intermittent vecuronium for neuromuscular blockade. Extubation on day 8 was unsuccessful because of quadriparesis, with diffuse muscle atrophy, and absent reflexes. Muscle strength gradually returned over 3 months, but hyporeflexia persisted for $>1$ year. (Sheth RD, Bodensteiner JB et al. Critical illness neuromuscular disease in children manifested as ventilatory dependence. I Pediatr February 1995;126:259-61). (Reprints: Raj D Sheth MD, West Virginia University Health Science Center, Box 9180, Morgantown, WV 26506).

COMMENT. Critical-illness polyneuropathy, a complication of sepsis in adults, and a cause of difficulty in weaning from the ventilator, is covered in Progress in Pediatric Neurology II, 1994, pp275-276. The syndrome appears to be unusual in children.

\section{MUSCULAR FATIGUE IN DUCHENNE DYSTROPHY}

The fatigability of the anterior tibial muscle in 11 boys with Duchenne muscular dystrophy (DMD) was compared to that of controls at the California Pacific Medical Center and the University of California, San Francisco. The force generation of dystrophic muscle and compound muscle action potential amplitude were lower and relaxation time of tetanus was longer in patients than in controls at rest. During exercise, maximum voluntary contraction was better sustained, suggesting less central fatigue in DMD patients than in controls. (Sharma KR, Mynhier MA, Miller RG. Muscular fatigue in Duchenne muscular dystrophy. Neurology February 1995;45:306-310). (Reprints: Dr Khema R Sharma, University of Miami, Dept of Neurology, 1501 NW 9th Ave, Miami, FL 33136). 